\title{
Development of multielemental imaging on semiconductor Compton telescope*
}

\author{
Shinji Motomura ${ }^{1}$, Yousuke Kanayama ${ }^{1,2}$, Hiromitsu Haba ${ }^{1,2}$, \\ Kaori Igarashi ${ }^{2}$, Yasuyoshi Watanabe ${ }^{1}$, and Shuichi Enomoto ${ }^{1,2, \ddagger}$ \\ ${ }^{1}$ Molecular Imaging Research Program, RIKEN, 6-7-3 Minatojima-minamimachi, \\ Chuo-ku, Kobe, Hyogo 650-0047, Japan; ${ }^{2}$ Metallomics Research Unit, RIKEN, \\ 2-1 Hirosawa, Wako, Saitama, 351-0198, Japan
}

\begin{abstract}
The feasibility of using a Compton camera for multitracer imaging has been demonstrated with the results of two biological sample imaging experiments. The distribution of the multitracer administered to a soybean sample and a tumor-bearing mouse has been visualized for each nuclide simultaneously. 3D images of the multitracer have been obtained even though the samples were measured from a fixed direction.
\end{abstract}

Keywords: Compton; Compton telescope; molecular imaging; multitracer imaging; Compton camera.

\section{INTRODUCTION}

The multitracer technique, which was invented at RIKEN in 1991 [1,2], is a powerful tool for investigating the behavior of various chemical elements in a sample. It is produced by irradiating a metal target with a heavy-ion beam accelerated to an energy of $135 \mathrm{MeV} / \mathrm{u}$, and then by chemically processing the various radioactive nuclides produced mainly through nuclear-fragmentation reaction, into the final form such as multitracer solution. Since the multitracer contains the radioisotopes of various elements, the information regarding the radioisotopes under the same conditions can be obtained simultaneously by a single experiment. Moreover, the multitracer enables us to observe the correlated behavior among many elements. This information can never be obtained by combining the data of many single-tracer experiments. Owing to these advantages, the multitracer has found many applications in biology, medicine, environmental science, and other fields [3].

Although the potential advantages of multitracers are promising, no nondestructive inspection method has yet been established to realize their full potential particularly for in vivo imaging. This is because the multiple $\gamma$-rays emitted from the multitracer span an energy range from $\sim 100$ to $2 \mathrm{MeV}$. For energies above $\sim 300 \mathrm{keV}$, sufficient spatial resolution cannot be obtained by a conventional $\gamma$-ray imager equipped with mechanical collimators. In addition, the energy resolution must be high enough to distinguish each nuclide contained in the multitracer.

In this paper, we describe a prototype of a Compton camera for multitracer imaging, which we call GREI (gamma-ray emission imaging), and present some results of test experiments with biological samples. The original idea of the Compton camera was invented in the early 1970s [4], and it was soon

\footnotetext{
*Paper based on a presentation at the International Symposium on Metallomics 2007 (ISM 2007), 28 November-1 December 2007, Nagoya, Japan. Other presentations are published in this issue, pp. 2565-2750.

¥Corresponding author
} 
proposed for medical imaging [5]. Since then, various types of Compton camera have been proposed for various uses [6].

Previously, we performed a test experiment using a Compton camera composed of two segmented germanium $(\mathrm{Ge})$ detectors [7]. Owing to the excellent energy resolution of the Ge detectors, three $\gamma$-ray sources of ${ }^{60} \mathrm{Co},{ }^{137} \mathrm{Cs}$, and ${ }^{152} \mathrm{Eu}$ were clearly distinguished by setting energy windows on the corresponding $\gamma$-ray photo peaks, and their positions were simultaneously determined. The GREI system described in this paper has been modified taking into account the results of the test experiment, further simulations [8], and recent technologies developed for $\gamma$-ray detection [9-14].

\section{DESCRIPTION OF THE GREI SYSTEM}

The GREI system is composed of two double-sided orthogonal-strip Ge detectors manufactured by Eurisys Mesures (Fig. 1). The detectors are arranged parallel to each other and mounted in a single cryostat. The dimensions of the active volume of the Ge crystals are $39 \times 39 \times 10 \mathrm{~mm}$ and $39 \times 39 \times$ $20 \mathrm{~mm}$ for the front and rear detectors, respectively. The strip pitch is $3 \mathrm{~mm}$ for both detectors. The center-to-center distance between the crystals is $60 \mathrm{~mm}$.

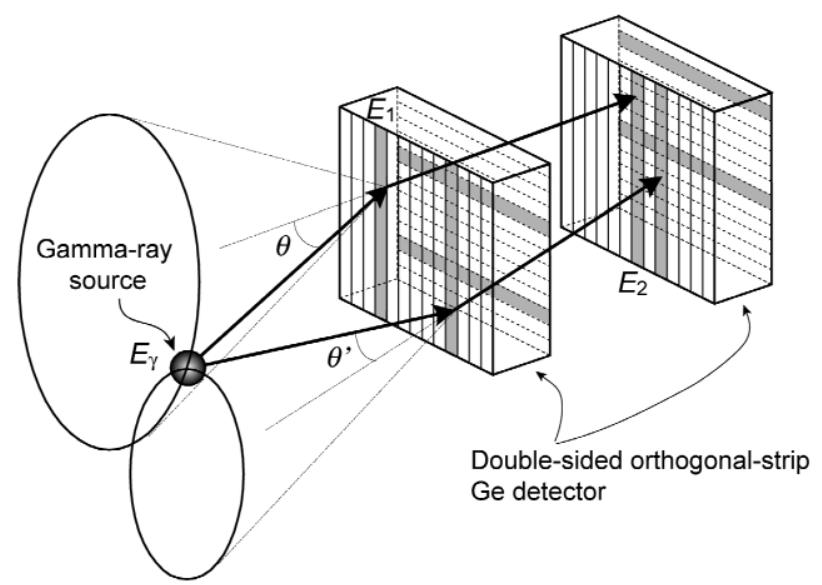

Fig. 1 Schematic of the strip Ge telescope operated as a Compton camera.

The electronics are schematically shown in Fig. 2. Conventional circuit modules based on nuclear instrument module (NIM) and computer automated measurement and control (CAMAC) standards are used. All the output signals of the preamplifiers are fed into the constant fraction discriminators (CFDs) through the timing filter amplifiers (TFAs), and the timing pulses are generated. All the timing pulses are fed into the time-to-digital converters (TDCs) to digitize the timing signals. To select the Compton scattering events, the timing pulses derived from the cathode strips are used to perform coincidence measurement between the front and rear detectors.

All the output signals of the preamplifiers are also fed into the shaping amplifiers to generate slow signals for energy measurement. Then the pulse heights are digitized by the analog-to-digital converters (ADCs).

In the current implementation, the transverse position of the $\gamma$-ray interaction is determined by a combination of the anode and cathode strips with the accuracy given by the width of the strip. On the other hand, the depth position of the $\gamma$-ray interaction in the Ge crystal can be determined more accurately than the thickness of the crystal, by taking into account the time difference between the signals from the anode and cathode strips [9-14]. To realize accurate depth measurement, the time constants of TFAs were set to 500 and $60 \mathrm{~ns}$ for differentiation and integration, respectively. Delay time 


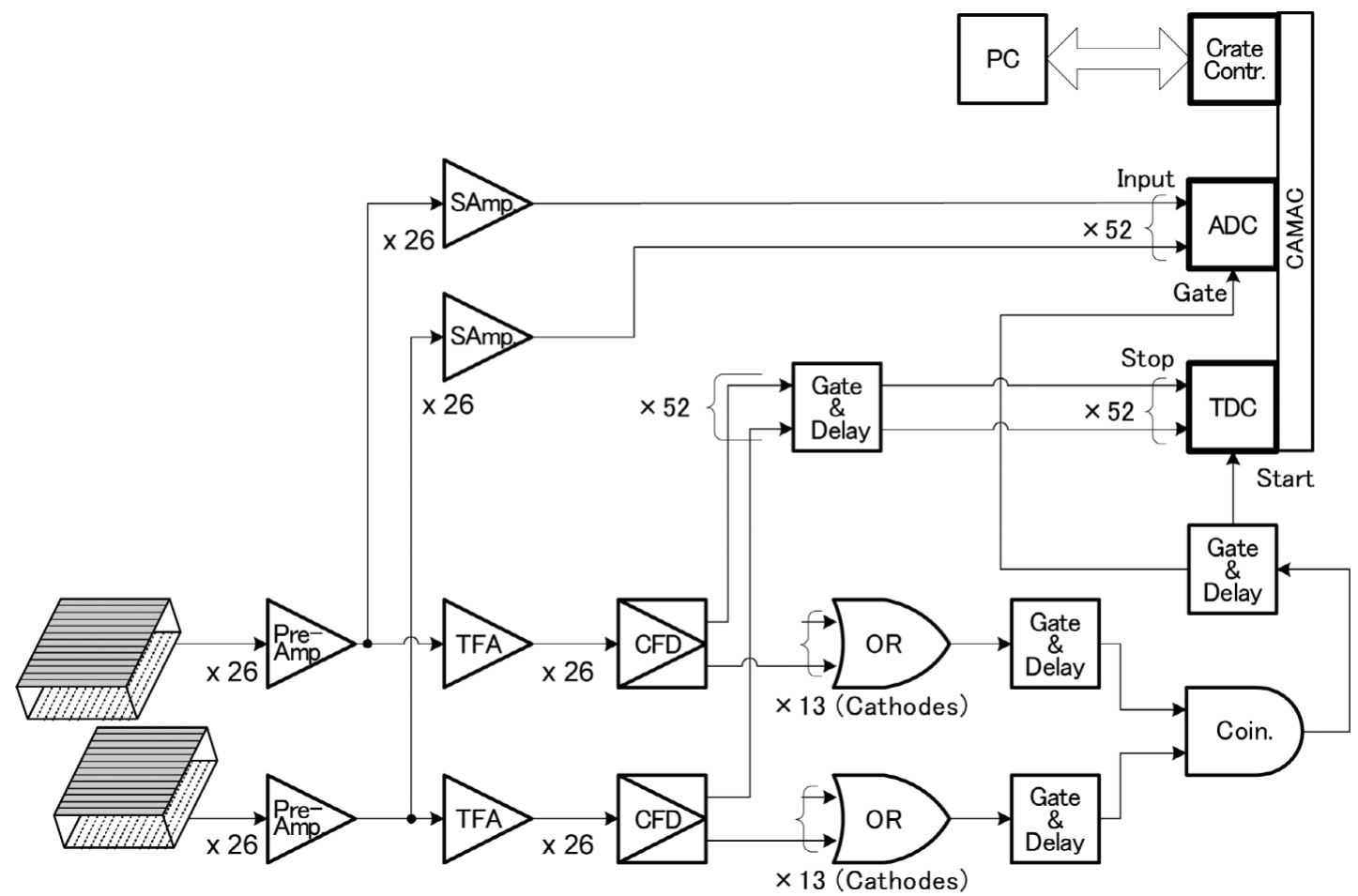

Fig. 2 Circuit diagram of the current prototype of the GREI system. The circuit modules are based on NIM and CAMAC standards.

of the CFDs was set to $50 \mathrm{~ns}$, which was the upper limit of the modules, and the fraction of the CFDs was set to 0.5 .

The digitized data of the TDCs and ADCs are transferred event by event to the personal computer. The transferred data are analyzed on-line, and also recorded in list mode for further off-line analysis. If an incident $\gamma$-ray is Compton scattered in the front detector and deposits energy $E_{1}$, and then the scattered $\gamma$-ray is fully absorbed in the rear detector and deposits energy $E_{2}$, the original $\gamma$-ray energy $E_{\gamma}$ is obtained by summing $E_{1}$ and $E_{2}$. Thus, the nuclides contained in the multitracer are distinguished by setting energy windows at the corresponding energy peaks in the $E_{\gamma}$ spectrum. Throughout this paper, the range of an energy window was $\pm 5 \mathrm{keV}$ of the peak position and the windows were applied in the software.

\section{IMPLEMENTATION OF IMAGE RECONSTRUCTION METHODS}

For image reconstruction, we are currently employing a two-step method. In the first step, an intermediate image, which is called a simple back-projection (SBP) image, is constructed by simply accumulating the back-projections over all detected events, using an algorithm analogous to the cone-surface mapping algorithm [15]. The back-projection for one event is a cone surface that indicates the possible position of the $\gamma$-ray source. The cone axis is the straight line passing through the first and second interaction points. The half-cone angle $\theta$ is the Compton scattering angle determined by the following equation: 


$$
\cos \theta=1+m_{\mathrm{e}} c^{2}\left(\frac{1}{E_{\gamma}}-\frac{1}{E_{\gamma}-E_{1}}\right)
$$

where $m_{\mathrm{e}} c^{2}$ is the rest-mass energy of an electron.

Here, we adopt a model that the SBP image is constructed by linear mapping of the source distribution image; that is,

$$
n_{i}=\sum_{j} p_{i j} \lambda_{j}
$$

where $n_{i}$ is the value of voxel $i$ in the SBP image, $\lambda_{j}$ is the value of voxel $j$ in the source distribution image, $p_{i j}$ is the point-spread function (PSF), or the point kernel function [15], which represents the SBP image of the point source at voxel $j$; the 3D coordinates are represented by the single indices $i$ and $j$. In general, the shape of the PSF varies depending on the source position, because the range of the accepted incident angle and scattering angle of $\gamma$-rays depends on the source position. Therefore, a spatially variant PSF must be used to perform rigorous image reconstruction. However, the PSF should be approximated by a spatially invariant PSF within a local region.

Then the second step is to deconvolve the SBP image with the PSF. We have implemented an analytical algorithm and an iterative algorithm for the deconvolution.

If the PSF is assumed to be spatially invariant, $\lambda_{j}$ can be reconstructed analytically by adopting the Fourier convolution theorem. The matrix $p_{i j}$ is diagonalized by taking the Fourier transform of both sides of eq. 2 , and then $\lambda_{j}$ is obtained in the spatial frequency domain as follows:

$$
\lambda_{j}=\frac{n_{j}}{p_{j j}}
$$

In most cases, an additional filter function $\left(w_{j}\right)$ must be multiplied to the right side of eq. 3 in order to suppress the statistical noise components, which are dominant in the high-frequency regions, that is

$$
\lambda_{j}=\frac{w_{j} n_{j}}{p_{j j}}
$$

If a Wiener filter function can be designed for the system, eq. 4 becomes the optimal estimation in terms of the least square errors. In addition, ad hoc filter functions, such as a Butterworth function, can be used for $w_{j}$ to suppress the noise components in the high-frequency regions.

We have also implemented an iterative deconvolution algorithm, which was adapted from the additive simultaneous reconstruction technique (SIRT) algorithm [16]. First, an initial estimate of the original image $\lambda_{j}^{(0)}$ is obtained by averaging the SBP image

$$
\lambda_{j}^{(0)}=\frac{1}{N} \sum_{i=1}^{N} n_{i}
$$

Then the $(n+1)$-th estimate $\left(\lambda_{j}^{(n+1)}\right)$ is obtained from the $n$-th estimate $\left(\lambda_{j}^{(n)}\right)$ as follows:

$$
\lambda_{j}^{(n+1)}=\lambda_{j}^{(n)}+\sum_{i}\left(n_{i}-\sum_{k} \lambda_{k}^{(n)} p_{i k}\right) p_{i j}
$$

Although spatially variant PSFs can be used for $p_{i j}$, spatially invariant PSFs have been used in the current work. 
A close examination shows that eq. 6 is the iteration term that minimizes the square errors of the SBP image constructed from the estimated source distribution. This can be seen if we recast the steepest descent iteration term from [17].

$$
\begin{aligned}
\Delta \lambda_{j}^{(n)} & =-\varepsilon \nabla \chi^{2}\left(\lambda_{j}^{(n)}\right) \\
& =-\varepsilon \frac{\sum_{i} \partial\left(n_{i}-\sum_{k} \lambda_{k}^{(n)} p_{i k}\right)^{2}}{\partial \lambda_{j}^{(n)}} \\
& =2 \varepsilon \sum_{i}\left(n_{i}-\sum_{k} \lambda_{k}^{(n)} p_{i k}\right) p_{i j}
\end{aligned}
$$

where $\Delta \lambda_{j}^{(n)}$ is the displacement of the estimate in the iteration step, $\varepsilon$ is a positive coefficient used to control the iteration. When $\varepsilon=0.5$, the second term on the right-hand side of eq. 6 is obtained.

Since the PSF has a 3D distribution, 3D deconvolution must be performed in order to reconstruct $3 \mathrm{D}$ images. However, when the source distribution can be considered to be $2 \mathrm{D}$, corresponding to the case of a sliced sample, 2D deconvolution is sufficient because there is no contamination from outside of the 2D plane. Thus, we have implemented both 2D and 3D deconvolution algorithms.

\section{PERFORMANCE OF THE PROTOTYPE}

The absolute efficiencies for obtaining the full-energy $\gamma$-ray peaks from the sources $15 \mathrm{~mm}$ away from the center of the front detector are shown in Fig. 3, together with the values estimated by Monte Carlo simulation using a GEANT (GEometry ANd Tracking) [18] code. The experimental values were derived from the experimental data mentioned in the next section. One can see that the GREI system has significant efficiency between the energy range from $\sim 200 \mathrm{keV}$ to $\sim 2 \mathrm{MeV}$.

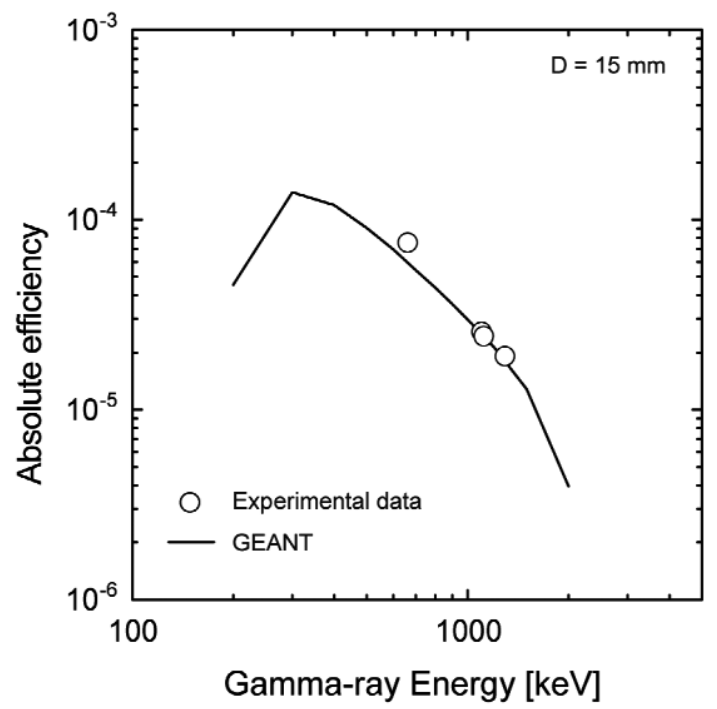

Fig. 3 Absolute efficiencies for obtaining the full-energy $\gamma$-ray peaks. The solid line indicates the numerical values calculated with a Monte Carlo simulation code GEANT [18]. 
The effect of the statistical noise on the spatial resolution achieved with the prototype system was investigated in terms of the Fourier power spectral density (FPSD) of the SBP image. A point-like source of ${ }^{65} \mathrm{Zn}$, which emits $1116 \mathrm{keV} \gamma$-rays, was placed $69 \mathrm{~mm}$ away from the center of the front detector, and then three SBP images were constructed in 3D space with the detected event numbers of $1.0 \times 10^{4}, 5.0 \times 10^{4}$, and $1.0 \times 10^{5}$. Then the SBP images were analytically deconvolved with the PSF constructed using a GEANT code. The full widths at half maximum (FWHMs) of the reconstructed images of the point source are shown in Table 1 for each number of events.

Table 1 Spatial resolution of the reconstructed image in FWHM, and derived transient spatial frequency $\left(f_{c}\right)$ of the FPSD of the SBP image, where the statistical noise components are beginning to dominate. These values were determined for three different numbers of detected Compton scattering events.

\begin{tabular}{lcccc}
\hline \multirow{2}{*}{$\begin{array}{l}\text { Number } \\
\text { of events }\end{array}$} & \multicolumn{2}{c}{ FWHM $[\mathrm{mm}]$} & \multicolumn{2}{c}{$f_{\mathrm{c}}\left[\mathrm{mm}^{-1}\right]$} \\
\cline { 2 - 5 } x-direction & z-direction & x-direction & z-direction \\
\hline $1.0 \times 10^{5}$ & 4.9 & 11.4 & $0.16 \pm 0.02$ & $0.06 \pm 0.01$ \\
$5.0 \times 10^{4}$ & 6.0 & 12.9 & $0.15 \pm 0.02$ & $0.05 \pm 0.01$ \\
$1.0 \times 10^{4}$ & 10.3 & 14.2 & $0.12 \pm 0.03$ & $0.04 \pm 0.01$ \\
\hline
\end{tabular}

\section{Experiment with biological samples}

We have performed experiments with two biological samples in order to demonstrate the capability of nondestructive imaging of a multitracer. The first sample was a soybean plant administered with $310-\mathrm{kBq}{ }^{137} \mathrm{Cs}, 89-\mathrm{kBq}{ }^{59} \mathrm{Fe}$, and $20-\mathrm{kBq}{ }^{65} \mathrm{Zn}$. A photograph of the sample is shown in Fig. 6 . The sample was fixed on a plane $15 \mathrm{~mm}$ away from the center of the front Ge detector. The measurement was carried out for $25 \mathrm{~h}$.

Figure 4 shows the results of 2D imaging of the soybean sample. The energy windows were set at each peak position indicated in the $\gamma$-ray energy spectrum (Fig. 4a) to distinguish the radionuclides of ${ }^{137} \mathrm{Cs},{ }^{59} \mathrm{Fe}$, and ${ }^{65} \mathrm{Zn}$. The 2D SBP images were constructed for each nuclide on the plane where the sample was fixed, assuming a 2D distribution, and then the analytical reconstruction method was used to deconvolve the 2D SBP images with the 2D PSFs. The resulting images successfully visualized the different behaviors of the nuclides. The ${ }^{137} \mathrm{Cs}$ nuclide was distributed throughout the whole of the sample, because it is chemically analogous to $\mathrm{K}$, while ${ }^{59} \mathrm{Fe}$ and ${ }^{65} \mathrm{Zn}$ remained near the root. The ${ }^{65} \mathrm{Zn}$ nuclide was found at the tip of the stem to some extent, where the plant was actively growing, because $\mathrm{Zn}$ is required for cell division.

In addition, we note that there is a difference in the background structure of the images. This is due to the difference in the number of events obtained for each nuclide; $2.5 \times 10^{6}, 3.4 \times 10^{5}$, and $7.3 \times$ $10^{4}$ for ${ }^{137} \mathrm{Cs},{ }^{59} \mathrm{Fe}$, and ${ }^{65} \mathrm{Zn}$, respectively. The smaller the number of events, the larger the statistical noise component becomes.

The second sample was a tumor-bearing mouse administered intravenously with a multitracer solution that includes $60-\mathrm{kBq}{ }^{65} \mathrm{Zn}, 30-\mathrm{kBq}{ }^{59} \mathrm{Fe}$, and $10-\mathrm{kBq}{ }^{88} \mathrm{Y}$. The sample was fixed on a board and placed just under the front detector, which was installed with its front face down. The measurement was carried out for $95 \mathrm{~h}$.

Figure 5 shows the results of $2 \mathrm{D}$ imaging of the tumor-bearing mouse. As in the case of the soybean sample, the energy windows were set at each peak position indicated in the $\gamma$-ray energy spectrum (Fig. 8a) to distinguish the radionuclides of ${ }^{65} \mathrm{Zn},{ }^{59} \mathrm{Fe}$, and ${ }^{88} \mathrm{Y}$. Then the $2 \mathrm{D}$ SBP images were constructed for each nuclide on the 2D plane that intersects the tumor part, and the SBP images were analytically deconvolved with the 2D PSFs. The resulting images are only "focused" images along the assumed plane, because the other parts of the sample have some contribution to the constructed 2D SBP 

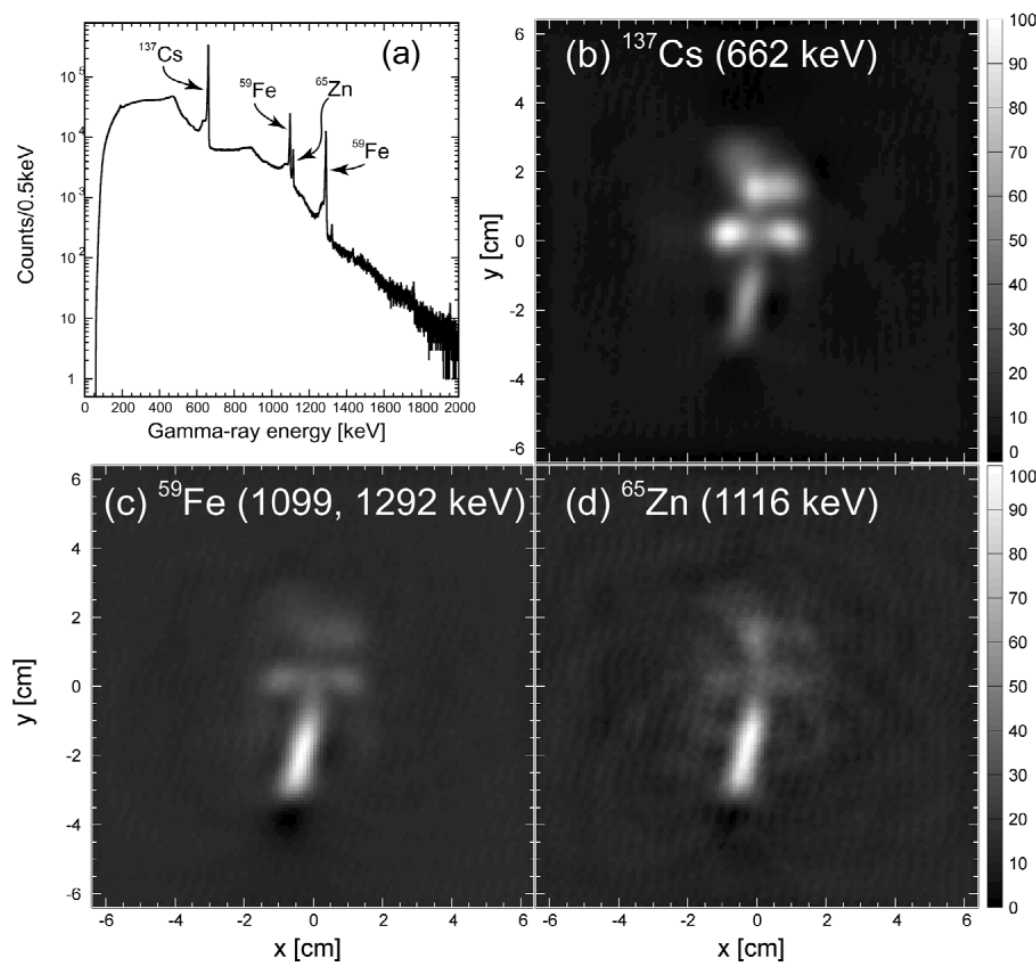

Fig. 4 Results of 2D imaging of the soybean sample.

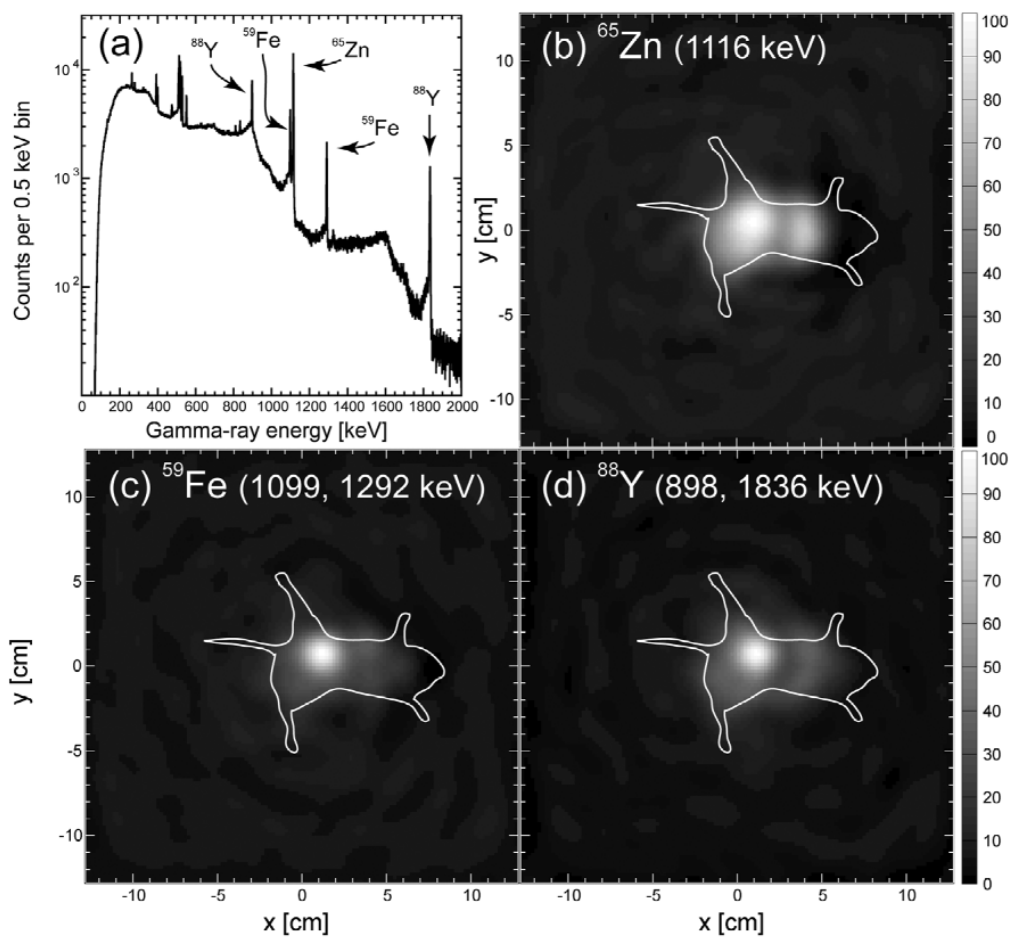

Fig. 5 Results of 2D imaging of the tumor-bearing mouse.

(C) 2008 IUPAC, Pure and Applied Chemistry 80, 2657-2666 
images. Nevertheless, the accumulation of the nuclides in the tumor site was evident and ${ }^{65} \mathrm{Zn}$ was also found in the liver. The results are consistent with those reported in [19-21].

We have performed 3D imaging of these samples, even though the samples were measured from a fixed direction. When the sample is sufficiently close to the front detector, the source distribution can be projected toward various directions because no mechanical collimator is used. This is a distinctive feature of a Compton camera. First, the 3D SBP images were constructed in 3D space, then the SBP images were deconvolved with the 3D PSFs.

Figure 6 shows the result of 3D imaging of ${ }^{137} \mathrm{Cs}$ in the soybean sample. The deconvolution was performed analytically. Since ${ }^{137} \mathrm{Cs}$ was distributed throughout the whole sample, the shape of the sample can be recognized. However, there exists a warp in the reconstructed image, which may have been caused by the use of spatially invariant PSFs.

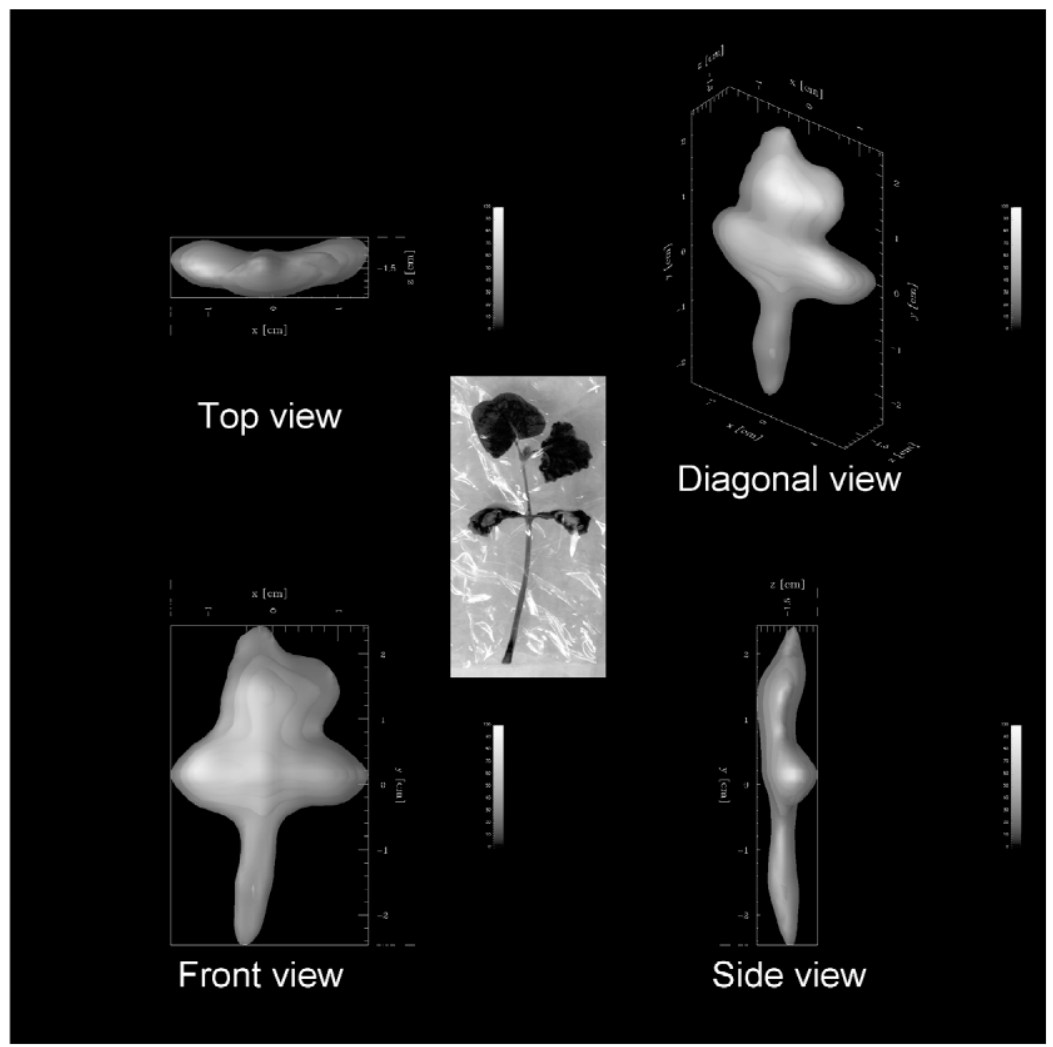

Fig. 6 Three-dimensional image of ${ }^{137} \mathrm{Cs}$ distributed in the soybean sample. A photograph of the sample is also shown at the center.

Figure 7 shows the result of 3D imaging of ${ }^{65} \mathrm{Zn}$ in the tumor-bearing mouse. In this case, we could not obtain any satisfactory images by analytical deconvolution. Thus, the reconstructed space was limited to only the region around the sample, and the iterative deconvolution was performed. The resulting image successfully visualized the accumulation of ${ }^{65} \mathrm{Zn}$ in the tumor and the liver. However, the image has a similar warp to the 3D image of the soybean sample. Spatially variant PSFs should be incorporated to obtain more accurate images.

We were able to demonstrate the feasibility of using the GREI system for nondestructive imaging of multitracer. Recently, we were succeeded in imaging dynamic metabolic processes of three radio- 


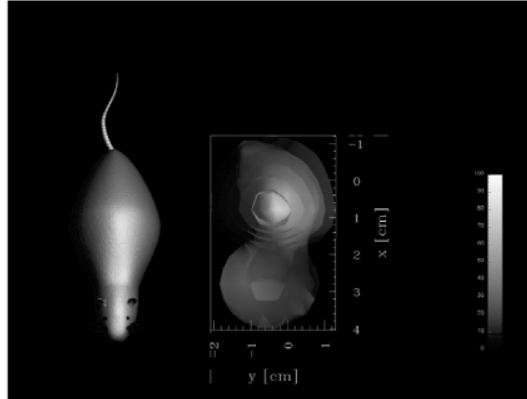

Top view

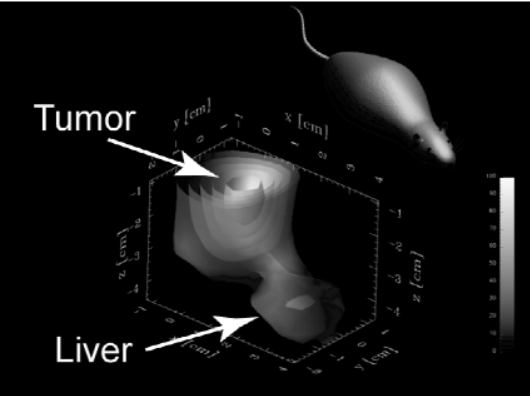

Diagonal view

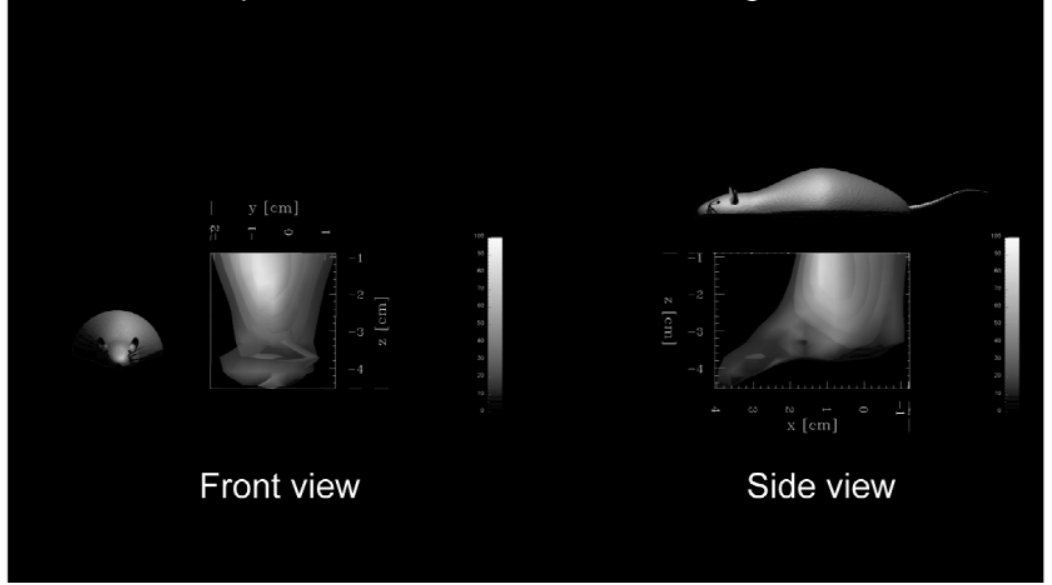

Fig. $73 \mathrm{D}$ image of ${ }^{65} \mathrm{Zn}$ distributed in the tumor-bearing mouse. The orientations of the sample are indicated by the mouse figures.

active medicines that were simultaneously administered [22]. However, the measurement times taken to obtain the images would be unsuitable for practical imaging, although they can be shortened to about $10 \mathrm{~h}$ if more intense $\gamma$-ray sources are used. Moreover, a higher spatial resolution would be desired to observe more fine structure. These demands would be met if the $\gamma$-ray tracking technique and pulseshape analysis are implemented, which have been recently developed [23]. With these techniques implemented, the efficiency and the intrinsic characteristics are improved, thereby shortening the measurement time and improving the spatial resolution.

\section{SUMMARY}

A prototype of a Compton camera, GREI, has been fabricated for nondestructive imaging of a multitracer. It is composed of two double-sided orthogonal-strip Ge detectors, and the efficiency is significant in the energy range from $\sim 200 \mathrm{keV}$ to $2 \mathrm{MeV}$. The timing method was used to derive the depth position of the $\gamma$-ray interaction with a resolution of $\sim 1 \mathrm{~mm}$ FWHM. Both analytical and iterative image reconstruction methods were implemented for $2 \mathrm{D}$ and $3 \mathrm{D}$ imaging. A test experiment was performed to demonstrate the capability of the GREI system. The resulting images successfully visualized the different behavior of each nuclide for both the soybean and the mouse sample. Furthermore, 3D images were obtained even though the samples were measured from a fixed direction. However, there was some distortion in the 3D images, which may have been caused by the use of spatially invariant PSFs. Spatially variant PSFs should be incorporated to obtain more accurate 3D images. To make the GREI 
system suitable for practical imaging, $\gamma$-ray tracking technique and pulse-shape analysis should be implemented.

\section{ACKNOWLEDGMENTS}

This work was supported by a Grant-in-Aid for Scientific Research of the Ministry of Health, Labour and Welfare of Japan and by a Grant for Nano Medicine Research of the New Enegy and Industrial Technology Development Organization of Japan. We thank Dr. R. Hirunuma and Dr. H. Takeichi for supporting the technical treatments.

\section{REFERENCES}

1. S. Ambe, S. Y. Chen, Y. Ohkubo, Y. Kobayashi, M. Iwamoto, M. Yanokura, F. Ambe. Chem. Lett. 20, 149 (1991).

2. H. Haba, D. Kaji, Y. Kanayama, K. Igarashi, S. Enomoto. Radiochim. Acta 93, 539 (2005).

3. S. Enomoto. Biomed. Res. Trace Elem. 16, 233 (2005).

4. V. Schönfelder, A. Hirner, K. Schneider. Nucl. Instrum. Methods 107, 385 (1973).

5. R. W. Todd, J. M. Nightingale, D. B. Everett. Nature 251, 132 (1974).

6. G. W. Phillips. Nucl. Instrum. Methods B 99, 674 (1995).

7. Y. F. Yang, Y. Gono, S. Motomura, S. Enomoto, Y. Yano. IEEE Trans. Nucl. Sci. 48, 656 (2001).

8. Y. F. Yang, Y. Gono, S. Motomura, S. Enomoto, Y. Yano. Nucl. Instrum. Methods A 482, 806 (2002).

9. M. Momayezi, W. K. Warburton, R. Kroeger. Proc. SPIE-Int. Soc. Opt. Eng. 3768, 530 (1999).

10. M. Amman, P. N. Luke. Nucl. Instrum. Methods A 452, 155 (2000).

11. E. A. Wulf, J. Ampe, W. N. Johnson, R. A. Kroeger, J. D. Kurfess, B. F. Phlips. IEEE Trans. Nucl. Sci. 49, 1876 (2002).

12. E. A. Wulf, B. F. Phlips, W. N. Johnson, R. A. Kroeger, J. D. Kurfess, E. I. Novikova. IEEE Trans. Nucl. Sci. 50, 1182 (2003).

13. S. Motomura, S. Enomoto, H. Haba, K. Igarashi, Y. Gono, Y. Yano. IEEE Trans. Nucl. Sci. 54, 710 (2007).

14. S. Kabuki, K. Hattori, R. Kohara, E. Kunieda, A. Kubo, H. Kubo, K. Miuchi, T. Nakahara, T. Nagayoshi, H. Nishimura, Y. Okada, R. Orito, H. Sekiya T. Shirahata, A. Takada, K. Ueno. Nucl. Instrum. Methods A 580, 1031 (2007).

15. R. C. Rohe, M. M. Sharfi, K. A. Kecevar, J. D. Valentine, C. Bonnerave. IEEE Trans. Nucl. Sci. 44, 2477 (1997).

16. P. Gilbert. J. Theor. Biol. 36, 105 (1972).

17. W. H. Press, S. A. Teukolsky, W. T. Vetterling, B. P. Flannery. Numerical Recipes in C: The Art of Scientific Computing, $2^{\text {nd }}$ ed., p. 681, Cambridge University Press, Cambridge (1992).

18. GEANT, Detector Description and Simulation Tool, CERN, Geneva (1993).

19. H. Tamano, S. Enomoto, B. Liu, A. Takeda. Biomed. Res. Trace Elem. 12, 96 (2001).

20. H. Tamano, S. Enomoto, N. Oku, A. Takeda. Nucl. Med. Biol. 29, 505 (2002).

21. A. Takeda, H. Tamano, S. Enomoto, N. Oku. Cancer Res. 61, 065 (2001).

22. S. Motomura, Y. Kanayama, H. Haba, Y. Watanabe, S. Enomoto. J. Anal. At. Spectrom. 23, 1089 (2008).

23. K. Vetter, M. Burks, L. Mihailescu. Nucl. Instrum. Methods Phys. Res., Sect. A 525, 322 (2004). 